\title{
Implante de Stent na Via de Saída de Ventrículo Direito: Alternativa à Paliação Cirúrgica Convencional?
}

\author{
Juliana Rodrigues Neves ${ }^{1}$, Raul Arrieta ${ }^{1}$, Renata Sá Cassar ${ }^{1}$, Cleusa Lapa $^{1}$, Catarina Cavalcanti ${ }^{2}$, \\ Ricardo Lins², Rodrigo Escobar², Alexandre Magno², Wagner Cid Cavalcante ${ }^{3}$
}

\section{RESUMO}

O uso de stents intravasculares em crianças com cardiopatia congênita é procedimento bem estabelecido, porém a indicação de paliação por meio de procedimentos percutâneos ao invés da cirurgia convencional permanece incerta. Neste relato são apresentados três casos de obstrução crítica de via de saída do ventrículo direito (VSVD), submetidos a ampliação da VSVD com implante de stent. O implante foi realizado com sucesso nos três casos, permitindo restabelecer o fluxo pulmonar para a árvore pulmonar e melhorar a saturação de oxigênio.

DESCRITORES: Obstrução do fluxo ventricular externo. Stents. Artéria pulmonar.

$\mathbf{N}$ a atualidade, o alívio cirúrgico de estenose infundibular em ventrículo direito, associada a cardiopatias congênitas como a tetralogia de Fallot, está associado a baixa mortalidade e morbidade. Quando o tratamento corretivo não é possível, em decorrência de hipoplasia intensa da árvore vascular pulmonar, a cirurgia paliativa do tipo shunt sistêmico-pulmonar é o tratamento de escolha. A ampliação cirúrgica da via de saída também pode ser uma alternativa em casos selecionados. Uma forma de paliação não-cirúrgica é desejável em situações como cardiopatias complexas ou variações anatômicas que dificultam a abordagem cirúrgica, hipóxia acentuada com acidose metabólica, neonatos e infantes que sabidamente necessitarão de novas

\footnotetext{
${ }^{1}$ Instituto de Medicina Integral Prof. Fernando Figueira (IMIP) - Recife, PE, Brasil.

2 Pronto-Socorro Cardiológico de Pernambuco (PROCAPE) - Recife, PE, Brasil.

${ }^{3}$ Universidade de Pernambuco (UPE) - Recife, PE, Brasil.

Correspondência: Juliana Rodrigues Neves. Instituto de Medicina Integral Prof. Fernando Figueira (IMIP) - Rua dos Coelhos, 300 - Boa Vista Recife, PE, Brasil - CEP 50070-550

E-mail: ju neves@uol.com.br

Recebido em: 18/2/2011 • Aceito em: 8/5/2011
}

ABSTRACT

Stenting the Right Ventricle Outflow Tract: Is It an Alternative to Palliative Surgery?

The use of intravascular stents in children with congenital heart disease is well established, however the use of palliative percutaneous therapy instead of a conventional surgery remains uncertain. We present three cases of critical right ventricular outflow tract (RVOT) obstruction treated with stenting to expand the RVOT. Stenting was successful in all three cases, reestablishing pulmonary flow into the pulmonary arteries and improving oxygen saturation.

KEY-WORDS: Ventricular outflow obstruction. Stents. Pulmonary artery.

abordagens cirúrgicas no futuro. $\mathrm{O}$ uso de stent tem sido amplamente descrito para alívio de diversas lesões vasculares, porém há pouca informação sobre seu uso para alívio de obstruções musculares no interior do ventrículo direito em cardiopatias congênitas.

\section{RELATO DO CASO}

Revimos três casos realizados consecutivamente, em laboratório de cateterismo cardíaco de centro de referência em cardiologia pediátrica, situado no Recife (PE, Brasil).

\section{Caso 1}

Paciente do sexo masculino, com 3 anos e 9 meses de idade, portador de tetralogia de Fallot com desconexão das artérias pulmonares, mostrou, em acompanhamento ambulatorial, piora da cianose e crises de hipóxia. O ecocardiograma evidenciou ausência de fluxo através da via de saída do ventrículo direito (VSVD) e foi difícil avaliar a patência do canal arterial; o paciente foi então encaminhado para o laboratório de cateterismo cardíaco. O cateterismo evidenciou canal arterial com bom fluxo para artéria pulmonar esquerda e estenose infundibular acentuada de ventrículo direito 
com fluxo intensamente reduzido para o tronco pulmonar (atresia pulmonar adquirida), não sendo visualizada a artéria pulmonar direita (Figura 1). A criança encontrava-se estável, porém com saturação de oxigênio $\left(\mathrm{SO}_{2}\right)$ em torno de $65 \%-70 \%$. Deciciu-se pela implantação de stent na VSVD. Através de cateter balloon wedge pressure $6 \mathrm{~F}$ (BBraun, Melsungen, Alemanha), em projeção lateral esquerda, foi ultrapassado infundíbulo com guia 0,014 polegada de angioplastia coronária. Nova injeção angiográfica detectou uma "terceira câmara" após a estenose infundibular. $\mathrm{O}$ anel pulmonar tinha dimensões conservadas, com discreto espessamento dos foIhetos valvares, e a artéria pulmonar direita era de bom calibre. O guia 0,014 polegada foi trocado por guia V18 de 0,018 polegada e $300 \mathrm{~cm}$ de comprimento (Boston Scientific Co., Natick, Estados Unidos) e através de bainha longa 7 F (Cook Medical Inc., Box Bloomington, Estados Unidos) foi implantado stent Express ${ }^{\mathrm{TM}} \mathrm{LD}$ Vascular (Boston Scientific Co.) 7 × 17 mm no infundíbulo, respeitando a válvula pulmonar. O procedimento foi realizado sem intercorrências e novas angiografias demonstraram nítida melhora de fluxo para tronco e artéria pulmonar direita. Houve melhora imediata de $\mathrm{SO}_{2}$ de $70 \%$ para $100 \%$ sob ventilação mecânica assistida. Pela possibilidade de hiperfluxo pulmonar à direita, optou-se por manter a ventilação mecânica por 12 horas. O paciente foi extubado sem intercorrências, recebendo alta da unidade de terapia intensiva (UTI) em 48 horas e alta hospitalar em 7 dias, em uso de furosemida $2 \mathrm{mg} / \mathrm{kg} / \mathrm{dia}$ e aspirina. Após 5 meses do implante o paciente permanece clinicamente bem, acianótico e aguardando definição de melhor opção cirúrgica.

\section{Caso 2}

Paciente do sexo masculino, com 4 anos e 7 meses de idade, portador de tetralogia de Fallot com má anatomia, evoluindo com piora progressiva da cianose nos últimos meses. O ecocardiograma evidenciou estenose pulmonar infundíbulo-valvar acentuada com hipoplasia moderada da árvore pulmonar, sendo encaminhado então para cateterismo cardíaco diagnóstico pré-operatório. Durante exame foi evidenciada estenose infundibular acentuada com hipofluxo pulmonar, e os ramos pulmonares apresentavam calibre conservado, porém com diminuição significativa do fluxo em seu interior. Após a angiografia, o paciente apresentou piora abrupta da $\mathrm{SO}_{2}$ para $20 \%$, sendo visualizada, por meio de angiografia manual, redução crítica do fluxo pulmonar por obstrução do infundíbulo. Foram realizadas todas as medidas clínicas para o tratamento da crise de hipóxia, porém sem resposta. Diante desse quadro grave e sem a possibilidade de encaminhar a criança para cirurgia de urgência, decidiu-se pela realização de implante de stent na VSVD. A técnica foi similar à descrita no caso anterior, sendo utilizado nesse paciente stent Express $^{\text {TM }}$ LD Vascular 7 × 19 mm (Figura 2). O procedimento foi realizado sem intercorrências e houve melhora imediata da $\mathrm{SO}_{2}(>90 \%)$ após o procedimento. Após estabilização clínica, o paciente foi extubado em sala de cateterismo e permaneceu na UTI por 12 horas, quando foi encaminhado ao hospital de origem para correção cirúrgica. A cirurgia foi realizada 10 dias após o procedimento, quando o stent foi retirado sem dificuldade técnica e foi feita ampliação da VSVD, com manutenção da valva pulmonar (Figura 3). Não foram registradas intercorrências no pós-operatório.

\section{Caso 3}

Paciente do sexo masculino, com 27 dias de vida, admitido em emergência pediátrica com quadro de dispneia e cianose acentuada. O paciente foi intubado

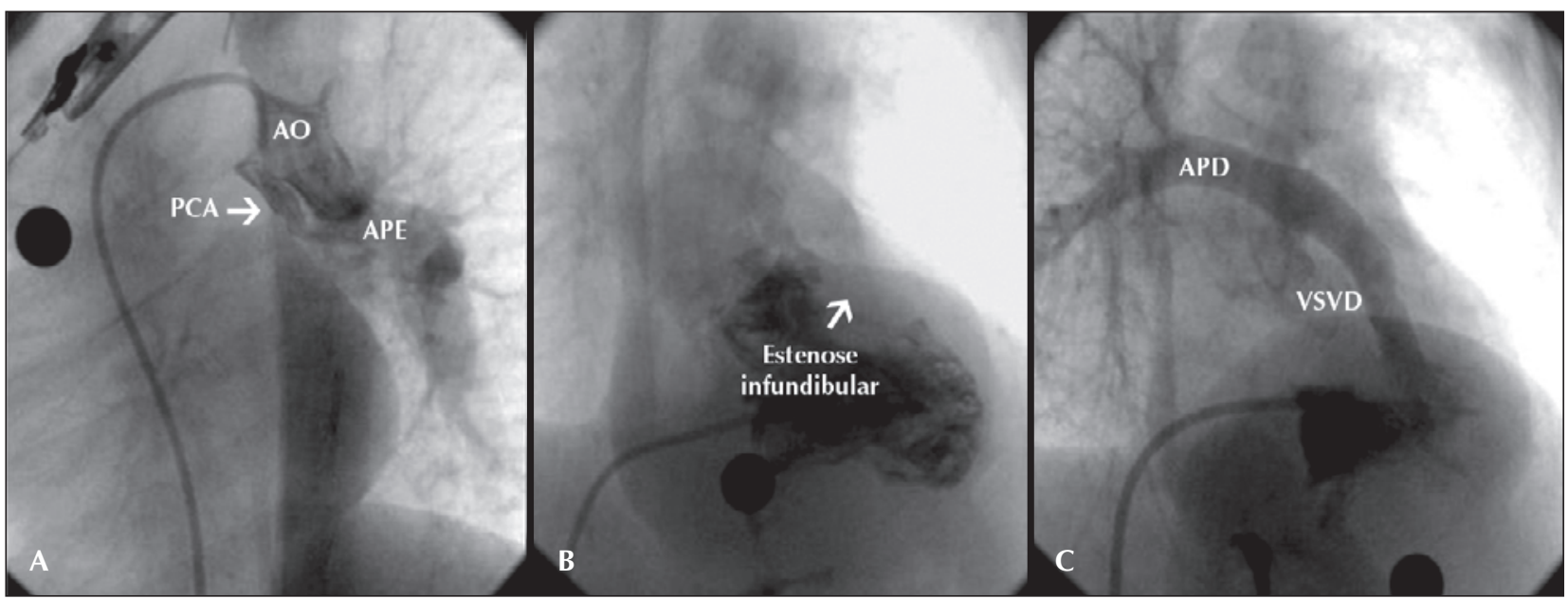

Figura 1 - Em A, injeção em aorta, mostrando persistência de canal arterial com manutenção de fluxo para artéria pulmonar esquerda. Em B, angiografia em ventrículo direito, demonstrando angustiamento acentuado de infundíbulo e virtual ausência de fluxo em artéria pulmonar. Em C, angiografia de controle após implante de stent, evidenciando bom fluxo através da via de saída do ventrículo direito, tronco da pulmonar e artéria pulmonar direita. $\mathrm{AO}=$ aorta; $\mathrm{APD}=$ artéria pulmonar direita; $\mathrm{APE}=$ artéria pulmonar esquerda; $\mathrm{PCA}=$ persistência do canal arterial; VSVD = via de saída do ventrículo direito. 


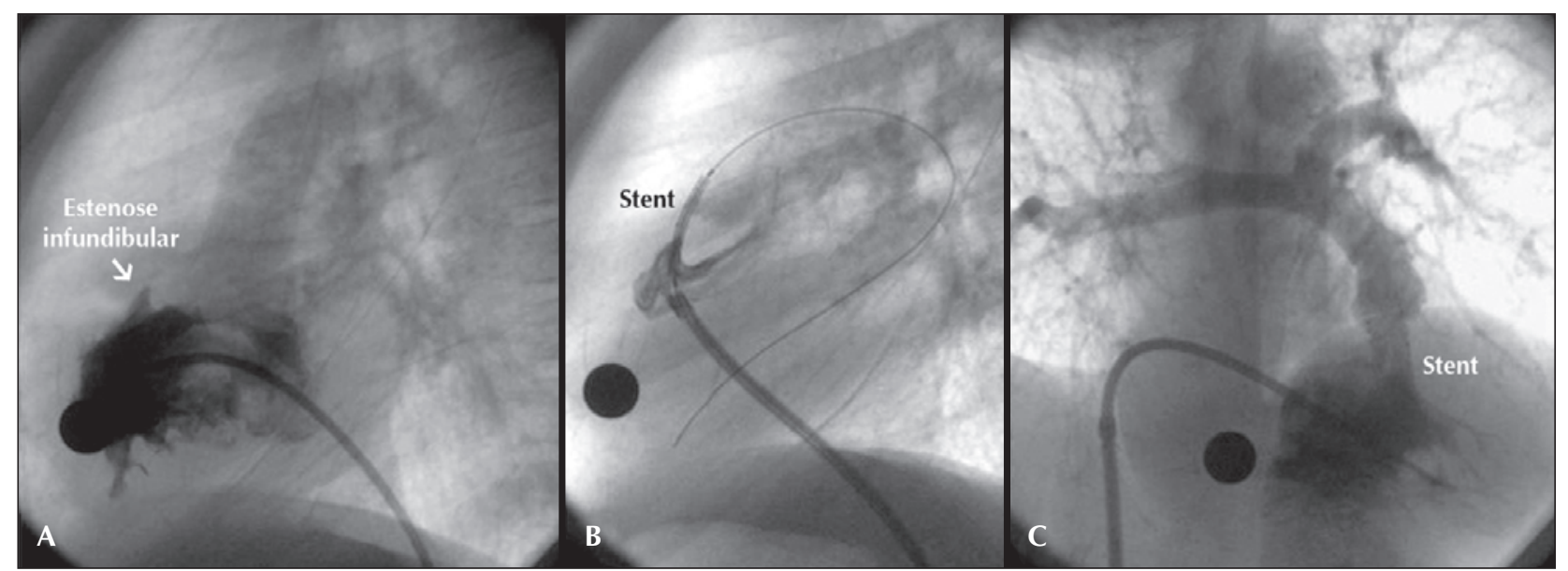

Figura 2 - Em A, injeção em ventrículo direito, demonstrando estenose infundibular grave. Em B, injeção através de bainha posicionada imediatamente antes da lesão, com stent posicionado, antes de sua liberação. Em C, angiografia de controle após a liberação do stent, demonstrando bom fluxo através do stent e adequada visualização de ramos pulmonares.

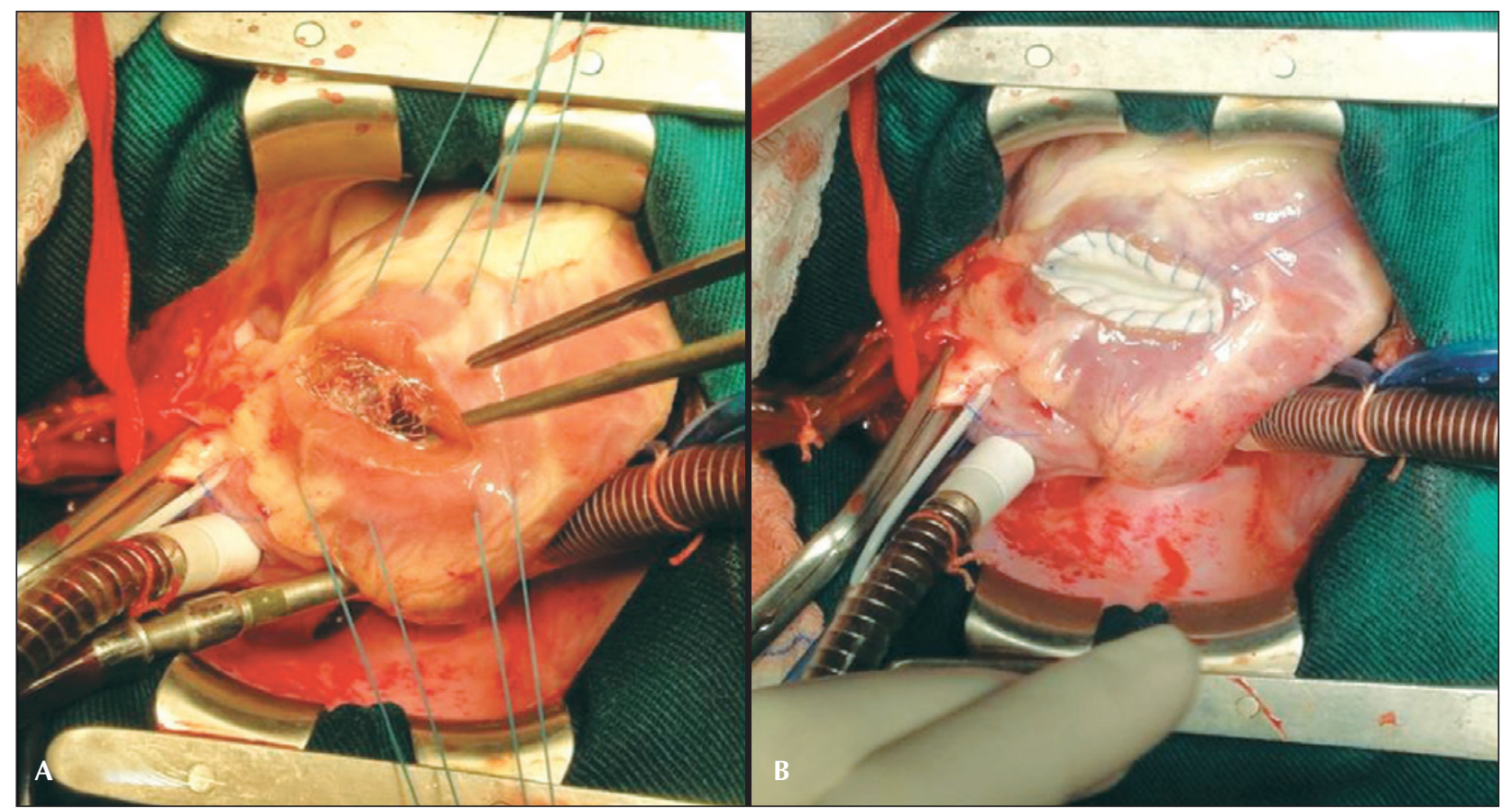

Figura 3 - Em A, stent sendo removido da via de saída do ventrículo direito, sem dificuldades técnicas. Em B, ampliação cirúrgica convencional, com preservação do anel pulmonar por meio de patch aplicado na via de saída do ventrículo direito.

por via orotraqueal e encaminhado à UTI, onde foi realizado ecocardiograma, que demonstrou atresia tricúspide IB (estenose infundibular acentuada, comunicação interventricular ampla) e mínimo canal arterial patente, localizado no arco aórtico (tipo vertical). Depois de tentado resgate de canal arterial com infusão intravenosa (IV) de prostaglandina, sem sucesso, a criança evoluiu com piora clínica progressiva, acidose metabólica refratária e $\mathrm{SO}_{2}<30 \%$, sendo encaminhada para tratamento percutâneo com implante de stent no canal arterial. Foram obtidos acesso venoso e acesso arterial femoral direito, sendo evidenciado, à aortografia, canal arterial fechado, sem possibilidade de passagem de guia 0,014 polegada. A ventriculografia esquerda em oblíqua anterior esquerda cranial demonstrou ampla comunicação interventricular, ventrículo direito com hipoplasia acentuada e estenose subvalvar pulmonar decorrente de estenose infundibular grave (Figura 4). Perante a situação clínica da criança, decidiu-se pela realização de implante de stent na porção infundibular do ventrículo direito. Através da veia femoral direita foi posicionado um cateter de coronária direita $4 \mathrm{~F}$ 
(Cordis Co., East Bridgewater, Estados Unidos) no interior do ventrículo esquerdo. Em seguida, a comunicação interventricular e o infundíbulo foram ultrapassados com guia 0,014 polegada de angioplastia coronária. Para dar melhor suporte ao implante do stent, um cateter de angioplastia coronária JR 6 F (Boston Scientific Co.) foi posicionado logo após a válvula mitral e um stent Liberté ${ }^{\mathrm{M}} 4$ × 15 mm (Boston Scientific Co.) foi implantado na VSVD. Durante a manobra de insuflação, a criança apresentou bradicardia e bloqueio atrioventricular 2:1, com resolução após o uso de adrenalina. Após nova angiografia, o infundíbulo encontrava-se totalmente pérvio, sem sinais de estenose e com melhora significativa do fluxo pulmonar. O quadro clínico melhorou acentuadamente logo após o procedimento, com aumento da $\mathrm{SO}_{2}$ para $85 \%$ e meIhora da acidose metabólica. O paciente persistiu intubado e sob ventilação mecânica por 72 horas, obtendo alta hospitalar após tratamento de quadro infeccioso associado. Em acompanhamento ambulatorial há 2 meses, o paciente apresentou $\mathrm{SO}_{2}$ de $75 \%$, encontrando-se em uso de ácido acetilsalicílico. O último ecocardiograma realizado evidenciou bom fluxo através do stent (gradiente de pico sistólico intrastent de
$16 \mathrm{mmHg}$ ), porém com gradiente significativo através da CIV de $40 \mathrm{mmHg}$.

Os dados dos pacientes encontram-se resumidos na Tabela.

\section{DISCUSSÃO}

Na maioria dos casos de obstrução crítica da VSVD, correção ou paliação cirúrgica têm sido realizadas com baixas taxas de morbidade e mortalidade em grandes centros. ${ }^{1}$ Entretanto, em pacientes com anatomia desfavorável, como hipoplasia ou distorção de artérias pulmonares, com comorbidades associadas ou criticamente enfermos, a possibilidade de paliação não-cirúrgica é desejável. O uso de stents em cardiopatias congênitas já foi amplamente descrito ${ }^{2,3}$, porém há poucos relatos de seu uso em obstruções musculares intracardíacas.

Os primeiros relatos do uso de stents em VSVD datam do início da década de 1990, como paliação para estenose infundibular isolada ou associada à tetralogia de Fallot e como alternativa à reconstrução da VSVD em pacientes com atresia pulmonar associada

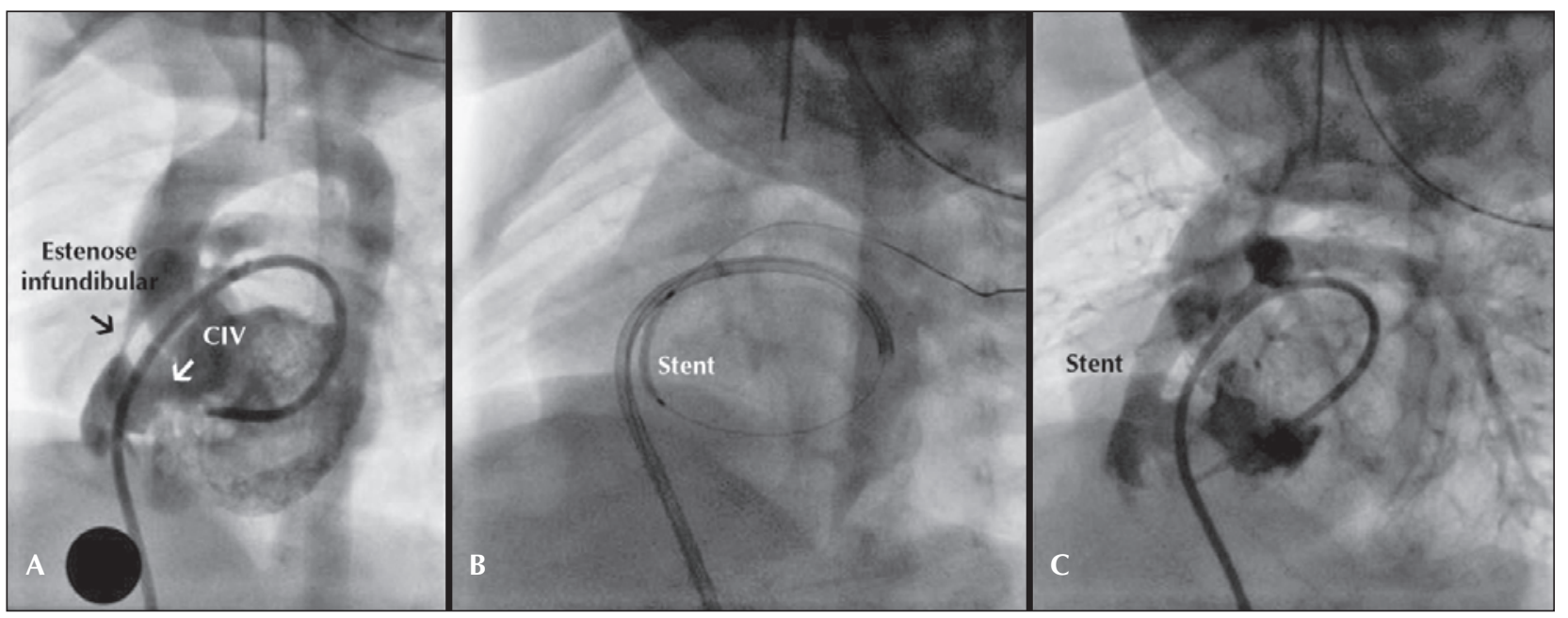

Figura 4 - Em A, ventriculografia esquerda em oblíqua anterior esquerda cranial, evidenciando fluxo satisfatório através da comunicação interventricular e significativo estreitamento em região infundibular. Em B, cateter-guia JR 6 F posicionado em átrio esquerdo e stent posicionado na altura da estenose. Em C, ventriculografia esquerda de controle após o procedimento, demonstrando bom fluxo através do stent. CIV = comunicação interventricular.

TABELA

Características dos pacientes submetidos a implante de stent em via de saída do ventrículo direito

\begin{tabular}{lccccccc}
\hline Caso & Idade & Diagnóstico & $\begin{array}{c}\mathrm{SO}_{2} \text { pré } \\
(\mathbf{\%})\end{array}$ & $\begin{array}{c}\mathrm{SO}_{2} \text { pós } \\
(\mathbf{\%})\end{array}$ & $\begin{array}{c}\text { UTI } \\
(\mathbf{d i a s})\end{array}$ & $\begin{array}{c}\text { Permanência } \\
\text { hospitalar (dias) }\end{array}$ & $\begin{array}{c}\text { Seguimento } \\
(\mathbf{d i a s})\end{array}$ \\
\hline 1 & 45 meses & T4F + exclusão APE & 72 & 100 & 1 & 7 & 188 \\
2 & 55 meses & T4F & 20 & 90 & 1 & $10^{*}$ & 10 \\
3 & 27 dias & Atresia tricúspide & $<20$ & 85 & 5 & 16 & 67 \\
\hline
\end{tabular}

* Até correção cirúrgica e remoção do stent.

$\mathrm{APE}=$ artéria pulmonar esquerda; $\mathrm{SO}_{2}=$ saturação de oxigênio; T4F = tetralogia de Fallot. 
a comunicação interventricular. ${ }^{4,5}$ Potenciais complicações dessa aplicação seriam a migração do stent, arritmias ventriculares, colapso ou fratura do stent, reestenose e hiperfluxo pulmonar.

Em nossa experiência inicial o implante de stent foi indicado em três casos como alternativa ao tratamento cirúrgico em pacientes com quadro clínico grave (crise de cianose), o que possibilitou a estabilização do paciente e, assim, a realização de programação cirúrgica em melhores condições clínicas.

Nos dois primeiros pacientes, portadores de tetralogia de Fallot, o stent foi implantado sem dificuldade técnica, porém alguns aspectos merecem ser ressaltados. A utilização de ventriculografia direita em perfil esquerdo possibilitou excelente visualização do local da estenose infundibular. Outro aspecto importante foi o uso do cateter-balão de furo terminal (pulmonary wedge catheter), que, pela sua flexibilidade, permitiu a orientação adequada para o infundíbulo e, assim, a ultrapassagem do guia 0,014 polegada. Nesse ponto, nova injeção possibilitou a correta visualização da "terceira câmara" e do anel valvar pulmonar, facilitando assim a medida do diâmetro da VSVD, fundamental para a escolha do tamanho e do comprimento do stent. Nos dois pacientes tratados, a valva pulmonar apresentava dimensões discretamente diminuídas e com discreto espessamento de seus folhetos, o que nos levou a optar pela colocação do stent apenas no local da estenose subvalvar, poupando a valva nativa. Nesse contexto, Dohlen et al. ${ }^{6}$ relatam a experiência com o uso de stents em VSVD como paliação de neonatos e lactentes jovens portadores de tetralogia de Fallot ou atresia pulmonar associada a comunicação interventricular com hipoplasia acentuada de artérias pulmonares, e enfatizam a necessidade de cobertura de todo o infundíbulo e da valva pulmonar, mesmo que para isso seja necessário o uso de stent muito longo ou de mais de um dispositivo. A manutenção da intubação após o procedimento é necessária pela possibilidade de hiperfluxo pulmonar, e não parece ser problema nesse grupo de pacientes, sendo necessário, em alguns casos, a terapia adicional com diuréticos por curto período de tempo. ${ }^{5,6}$

No caso do neonato de 27 dias de vida com atresia tricúspide IB, a indicação foi uma medida salvadora, já que esse paciente se encontrava em situação crítica. Esse tipo de procedimento, no entanto, pode não ser sempre factível, como no caso em questão, que se tratava de uma exceção em que a restrição ao fluxo pulmonar era infundibular e não relacionada à valva pulmonar ou à comunicação interventricular, como é habitual nessa afecção. Do ponto de vista técnico, ressaltamos dois pontos: o primeiro é a passagem do guia 0,014 polegada através da comunicação interventricular e do infundíbulo, auxiliado pelo posicionamento de um cateter de coronária direita logo após a valva mitral; o segundo foi o suporte adequado para o stent, utilizando um cateter-guia $6 \mathrm{~F}$, o que possibilitou boa sustentação da prótese no momento de sua passagem pelo infundíbulo.

Como complicação, nesse caso, observou-se bloqueio atrioventricular 2:1 com bradicardia acentuada revertida rapidamente com fármacos. A reversão rápida do bloqueio afasta a possibilidade de compressão mecânica do tecido de condução pelo stent, podendo ter ocorrido sua compressão temporária pelo balão de liberação ou justificado por se tratar de paciente criticamente enfermo em hipóxia e acidose acentuada, com obstrução temporária do fluxo pulmonar.

Apesar do curto período de seguimento desses pacientes, não foi observada reestenose dos stents. Os mecanismos de obstrução, na maioria dos casos, estão relacionados à obstrução muscular nas extremidades do stent, pela progressão de hipertrofia muscular na evolução tardia. ${ }^{5}$

O implante de stent em VSVD parece ser o modo de paliação alternativo em pacientes com obstrução ao fluxo pulmonar associado a cardiopatias congênitas, até mesmo a médio e longo prazos, quando há obstrução muscular isolada ou em tetralogia de Fallot com hipoplasia acentuada de artérias pulmonares. ${ }^{7,8}$ Nesses pacientes, quando submetidos a correção cirúrgica definitiva, a remoção do stent não parece comprometer o procedimento $^{6,9}$, como observamos em um caso. Apesar de nossos casos terem sido realizados em caráter de urgência, consideramos o implante de stent em VSVD alternativa possível à paliação cirúrgica convencional em pacientes selecionados.

\section{CONFLITO DE INTERESSES}

Os autores declaram não haver conflito de interesses relacionado a este manuscrito.

\section{REFERÊNCIAS}

1. Korbmacher B, Heusch A, Sunderdiek U, Gams E, Rammos $S$, Langenbach $M R$, et al. Evidence for palliative enlargement of the right ventricular outflow tract in severe tetralogy of Fallot. Eur J Cardiothorac Surg. 2005;27(6):945-8.

2. Fogelman R, Nykanen D, Smallhom JF, McCrindle BW, Freedom RM, Benson LN. Endovascular stents in the pulmonary circulation. Clinical impact on management and medium-term follow-up. Circulation. 1995;92(4):881-5.

3. O'Laughlin MP, Slack MC, Grifka RG, Perry SB, Lock JE, Mullins CE. Implantation and intermediate-term follow-up of stents in congenital heart disease. Circulation. 1993;88(2): 605-14.

4. Hausdorf G, Schulze-Neick I, Lange PE. Radiofrequencyassisted "reconstruction" of the right ventricular outflow tract in muscular pulmonary atresia with ventricular septal defect. Br Heart J. 1993;69(4):343-6.

5. Gibbs JL, Uzun O, Blackburn ME, Parsons JM, Dickinson DF. Right ventricular outflow stent implantation: an alternative to palliative surgical relief of infundibular pulmonary stenosis. Heart. 1997;77(2):176-9.

6. Dohlen G, Chaturvedi RR, Benson LN, Ozawa A, Van Arsdell GS, Fruitman DS, et al. Stenting of the right ventricular 
outflow tract in the symptomatic infant with tetralogy of Fallot. Heart. 2009;95(2):142-7.

7. Vincent RN, Diehl HJ. Unusual stents in infants with severe congenital heart disease. J Interv Cardiol. 2003;16(2):189-91.

8. Dryzek P, Mazurek-Kula A, Moszura T, Sysa A. Right ventricle outflow tract stenting as a method of palliative treat- ment of severe tetralogy of Fallot. Cardiol J. 2008;15(4): 376-9.

9. Laudito A, Bandisode VM, Lucas JF, Radtke WA, Adamson WT, Bradley SM. Right ventricular outflow tract stent as a bridge to surgery in a premature infant with tetralogy of Fallot. Ann Thorac Surg. 2006;81(2):744-6. 